\title{
EDITORIAL
}

\section{Cause and effect}

\author{
Identifying the causative agent of an infectious disease is a notoriously tricky, but vitally \\ important, business. When faced with new potential pathogens, the principles fostered by \\ Koch's postulates are as relevant today as they were over a century ago.
}

All microbiologists interested in infectious diseases are aware of the various statistics used to emphasize the threat microbial infection poses to human health, statistics that are regurgitated daily for conference audiences, funding bodies and journalists. One particularly interesting statistic, however, states that new infectious diseases are estimated to be emerging at the rate of one per year. In light of the potential ramifications of this statistic for global public health, a relevant question that requires consideration is: what constitutes a new infectious disease?

In the early days of microbiology, the attention of scientists and clinicians was primarily focused on diseases that presented with 'textbook' clinical symptoms and signs, that is, easily recognizable and distinguishable conditions. Many of the infectious diseases that preoccupy today's practitioners do not possess such obvious hallmark symptoms or signs. Patients can remain asymptomatic for decades, but these individuals act as reservoirs for some of our most deadly infections. Modern clinical advances, including transplantationrelated immunosuppression, have blurred the distinction between commensals and pathogens, and this fuzziness prevents a consensus being reached about what actually constitutes a pathogen ${ }^{1}$. These issues aside, more than 120 years after they were first proposed, Koch's postulates still remain the gold standard for any investigation that sets out to prove the aetiology of an infectious disease. Although advances in microbiology since the nineteenth century have led to modern reintepretations ${ }^{2,3}$, Koch's postulates, and the scientific rigour they foster, still provide an invaluable service in efforts to prove that a microorganism is the cause of a disease.

Take a recent case in point. Patients with chronic granulomatous disease (CGD) are subject to recurrent infections with a range of bacterial and fungal pathogens. In many cases, however, patients present with clinical symptoms that are indicative of an infection for which no obvious pathogen can be identified. In these cases, is disease caused by a known microorganism that eludes detection, or is a novel pathogen to blame? A recent study published in PLoS Pathogens ${ }^{4}$ describes the story of one CGD patient suffering from recurring lymphadenitis of unknown aetiology. The paper reports on the isolation and characterization of a new bacterium,
Granulobacter bethesdensis, from the lymph nodes of this patient. More importantly, by applying Koch's postulates with commendable rigour, the authors were able to convincingly establish a causal link between this novel organism and the infection in the patient - the first reported case of an invasive human disease caused by the Acetobacteraceae.

Another recent example provides more food for thought. Infection of the root canals of human teeth (commonly polymicrobial in nature), can lead to inflammation and the destruction of the periradicular tissues. A paper recently published in the Journal of Clinical Microbiology reports, for the first time, the detection and identification of an archaeal phylotype in a proportion of infected root canals ${ }^{5}$. This is an intriguing finding because although Archaea are recognized as a component of the human microbiota, it is generally assumed that this domain does not cause human disease. In this example, however, does an association of Archaea with an infected site prove that these organisms are actively contributing to disease? Are any of Koch's postulates fulfilled? By demonstrating an association between Archaea and infection, these findings certainly support a hypothesis that members of this domain can be human pathogens. An association, however, represents only one step on the journey to proof, and more research will be required before causality can be conclusively established in this case.

In the fight against newly emerging infectious diseases, the accurate identification of the offending aetiological agent will be essential if preventative and therapeutic measures are to be implemented effectively. It will serve the microbiological community well if Koch's postulates remain the gold standard to aim for when determining microbial cause and effect.

\footnotetext{
1. Falkow, S. What is a pathogen? ASM News 63, 359-365 (1997).

2. Falkow, S. Molecular Koch's postulates applied to bacterial pathogenicty - a personal recollection 15 years later. Nature Rev. Microbiol. 2, 67-72 (2004).

3. Fredericks, D. N. \& Relman, D. A. Sequence-based identification of microbial pathogens: a reconsideration of Koch's postulates. Clin. Microbiol. Rev. 9, 18-33 (1996).

4. Greenberg, D. E. et al. A novel bacterium associated with lymphadenitis in a patient with chronic granulomatous disease. PLOS Pathogens 2, e28 (2006).

5. Vianna, M. E. et al. Identification and quantification of archaea involved in primary endodontic infections. J. Clin. Microbiol. 44, 1274-1282 (2006).
} 